\title{
MUSEO DE LA PLATA RADIOCARBON MEASUREMENTS I
}

\section{ANIBAL FIGINI, GABRIEL GOMEZ, JORGE CARBONARI, ROBERTO HUARTE, and ALICIA ZUBIAGA}

Laboratorio de Tritio y Radiocarbono, Facultad de Ciencias Naturales y Museo, Paseo del Bosque, 1900 La Plata, Argentina

\section{INTRODUCTION}

The reinstallment, operation, and the ${ }^{14} \mathrm{C}$ measurements reported here were made since 1976 in the LATYR, the Radiocarbon Dating Laboratory of the Facultad de Ciencias Naturales y Museo, Universidad Nacional de La Plata. Sample preparation is preceded by careful visual separation and hand removal of gross impurities. This is followed in all cases (charcoal, wood) with successive washings of the sample with boiling $2 \% \mathrm{HCl}$ and $1 \% \mathrm{NaOH}$ solution for removal of carbonates and humic acids. Thereafter, it is washed with distilled water and acidified to $\mathrm{pH}=$ 3. Individual variations in the pretreatment are not described in the date list, but they are usually reported directly to the collector together with the data obtained.

Shell is dissolved in two fractions by HCl. The outer part of the shell (20\% in weight) is discarded to reduce possible surface contamination. The inner part (20 to $100 \%$ in weight) is used for dating. In some cases these two fractions are dated separately. Bone is pretreated by solution of collagen in acid hot water, according to Longin $(1970 ; 1971)$ with slight modifications. The chemical procedure for the groundwater samples preparation is to precipitate the carbon already in the field as barium carbonate. Atmospheric samples are collected by weekly static absorption in sodium hydroxide solution. In the lab, the solutions are converted to carbon dioxide by acid hydrolysis.

For organic materials the $\mathrm{CO}_{2}$ is produced in a tube combustion unit with an oxygen stream, with initial purification by $\mathrm{CuO}\left(600^{\circ} \mathrm{C}\right)$, acid potassium permanganate, and precipitation in sodium hydroxide solution in the form of barium carbonate. The solution is acid hydrolyzed in vacuo using phosphoric acid.

$\mathrm{CO}_{2}$ is evolved from carbonate samples by means of acid hydrolysis. We obtain the purest gases by final purification through a vacuum line with a water vapor trap $\left(-80^{\circ} \mathrm{C}\right)$, Cu furnace $\left(450^{\circ} \mathrm{C}\right)$, water vapor trap, and liquid air trap for $\mathrm{CO}_{2}$. Then, it is stored for three weeks for complete decay of radon.

Radioactivity is measured with a gas proportional counter, using carbon dioxide at $820 \mathrm{~mm} \mathrm{Hg}$ as filling gas, at room temperature $\left(20^{\circ} \mathrm{C}\right)$. The dating system has a $381 \mathrm{ml}$ copper-walled proportional counter, surrounded by $3 \mathrm{~cm}$ of selected lead, which is encircled by a ring of 14 cosmic-ray Geiger counters. Working voltage is $5200 \mathrm{v}$ with a plateau length of $300 \mathrm{v}$ and a plateau slope of ca $1 \%$ per $100 \mathrm{v}$ (for mesons).

Influence of cosmic and local environmental radiation on the counters is reduced with a composite shield consisting of layers of iron $(10 \mathrm{~cm})$, 
paraffin $(15 \mathrm{~cm})$, and iron $(30 \mathrm{~cm})$, at the top. All samples are counted at least twice for periods of not less than 2000 minutes each.

Age calculations are based on a contemporary value equal to 0.950 the specific activity of NBS oxalic acid standard and on the conventional Libby half-life for ${ }^{14} \mathrm{C}$ of $5570 \pm 30$ years. Results are reported in years before 1950 (years BP). Errors quoted $(l \sigma)$ include standard deviations of count rates for the unknown sample, contemporary standard, and background. Background $\mathrm{CO}_{2}$ is obtained from the acidification of marble and combustion of petroleum coke. The modern standard is prepared from NBS oxalic acid (SRM 4990 B) by wet oxidation with great care to achieve complete reaction (Valastro, Land, \& Varela, 1977; 1979). No corrections were made for isotopic fractionation and/or reservoir effect.

The description of each sample is based on information provided by the submitter.

ACKNOWLEDGMENTS

Financial support from the Consejo Nacional de Investigaciones Científicas y Técnicas (CONICET) of Argentina is gratefully acknowledged, and we would like to thank O Salvigsen and $\mathrm{H} S$ Jansen for providing inter-laboratory check samples. We also express our special recognition to Facultad de Ciencias Naturales y Museo, Universidad Nacional de La Plata, Argentina.

INTER-LABORATORY CHECK SAMPLES

\begin{tabular}{ccccc}
\hline Lab no. & LP date & Other lab no. & Other date & Refs \\
\hline LP-71 & $9910 \pm 240$ & T-2917 & $9580 \pm 150$ & Pers commun \\
LP-72 & $9280 \pm 250$ & T-2919 & $9480 \pm 140$ & Pers commun \\
LP-73 & $7460 \pm 210$ & R-9048* & $7670 \pm 70$ & Pers commun \\
\hline
\end{tabular}

* Internal no.-New Zealand lab

General Comment: apparent differences are considered statistically insignificant (Ward \& Wilson, 1978) and LP dates are in statistical concordance with those provided by other labs.

\title{
SAMPLE DESCRIPTIONS
}

\author{
GEOLOGIC SAMPLES
}

\section{Las Escobas I series}

\section{Argentina}

Marine coastal deposits corresponding to Las Escobas Fm, Prov Buenos Aires. Coll and subm by F Fidalgo, Fac Sci Nat Mus (FCNyM), Univ Nac La Plata (UNLP).

LP.7. Rincón Grande

Pelecypod shells from Rincón Grande ( $35^{\circ} 49^{\prime} 28^{\prime \prime} \mathrm{S}, 57^{\circ} 29^{\prime} 20^{\prime \prime} \mathrm{W}$ ), Partido de Castelli, depth $0.9 \mathrm{~m}$. 
LP.8. Rincón de López, Sample 1

$3050 \pm 160$

Pelecypod shells from Rincón de López ( $35^{\circ} 47^{\prime} 08^{\prime \prime}$ S, $\left.57^{\circ} 24^{\prime} 20^{\prime \prime} \mathrm{W}\right)$,

Partido de Castelli, depth 1.2m.

LP.9. Canal 18

$\mathbf{5 7 7 0} \pm \mathbf{1 7 0}$

Pelecypod shells from exposed profile near Estancia San Horacio $\left(35^{\circ}\right.$ $42^{\prime} 51^{\prime \prime} \mathrm{S}, 57^{\circ} 35^{\prime} 30^{\prime \prime} \mathrm{W}$ ), partido de Chascomús, depth $0.7 \mathrm{~m}$.

General Comment: results confirm Holocene age of Las Escobas Fm (Fidalgo, 1979).

\section{Bahia San Antonio series}

Marine shells coll from Baliza San Matías and San Antonio Fms (Angulo et al, 1978; Fidalgo et al, 1980), Bahía de San Antonio, Prov Río Negro. Both deposits record marine advances during Upper Pleistocene and Holocene times, respectively. Mollusk shells were taxonomically determined by Z Castellanos (FCNyM, UNLP). Coll and subm by F Fidalgo.

LP-33. Baliza San Matías

$>27,000$

Shells (Crepidula dilatata) $\left(40^{\circ} 49^{\prime} 22^{\prime \prime}\right.$ S, $\left.64^{\circ} 45^{\prime} 17^{\prime \prime} \mathrm{W}\right)$, Baliza San Matías Fm. Comment: inner fraction $(80 \%)$ was used.

LP-35. San Antonio, Sample 1

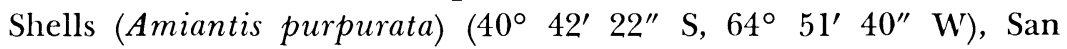
Antonio Fm. Comment: inner fraction $(80 \%)$ was used.

LP.36. Ruta no. 3

Shells (Glycimeris longior) $\left(40^{\circ} 44^{\prime} 35^{\prime \prime} \mathrm{S}, 65^{\circ} 02^{\prime} 30^{\prime \prime} \mathrm{W}\right)$, San Antonio Fm. Comment: inner fraction $(80 \%)$ was used.

LP-44. San Antonio, Sample 2

Shells (Clamys sp) $\left(40^{\circ} 42^{\prime} 22^{\prime \prime} \mathrm{S}, 64^{\circ} 51^{\prime} 40^{\prime \prime} \mathrm{W}\right)$, San Antonio Fm. Comment: inner fraction $(80 \%)$ was used.

LP-45. Caleta Falsa

Shells (Chionidae sp) $\left(40^{\circ} 47^{\prime} 56^{\prime \prime} \mathrm{S}, 64^{\circ} 50^{\prime} 38^{\prime \prime} \mathrm{W}\right)$, Baliza San Matías Fm. Comment: inner fraction $(80 \%)$ was used.

General Comment: geologically, difference observed between ${ }^{14} \mathrm{C}$ ages and relative age assigned to San Antonio Fm deposits would indicate that mollusk specimens might have been retransported from older units. Min ages obtained ( $3 \sigma$ criterion) are related to pelecypod ages and not deposit age (Fidalgo et al, 1980).

\section{Las Escobas II series}

Samples were coll from Destacamento Río Salado and Las Escobas Fms, Prov Buenos Aires. First lithostratigraphic unit could have developed during last glaciation, and other unit during Holocene (Fidalgo, 1979). Samples coll by F Fidalgo. Mollusk shells were coll by LP lab (Fidalgo et al, 1981). Bone was taxonomically id. by E Tonni (FCNyM, UNLP). Mollusk shells were id. by Z Castellanos (FCNyM, UNLP). X-ray diffraction analyses were made by M Iñíguez Rodríguez (FCNyM, UNLP). 
LP.46. Villa Catella

$4730 \pm 220$

Collagen extracted from the mandibular branch fragment of Eubalaena sp, Villa Catella ( $34^{\circ} 53^{\prime} 39^{\prime \prime}$ S, $57^{\circ} 56^{\prime} 47^{\prime \prime}$ W), Partido de Ensenada, from within sediments of ancient shoreline pertaining to Las Escobas Fm.

LP-47. Villa Catella

$3470 \pm 230$

Bone apatite extracted following Haynes (1968) from same mandibular branch fragment of Eubalaena sp; thus, same sample as LP-46.

\section{LP.49. Rincón de López, Sample 2}

$\mathbf{7 0 3 0} \pm 320$

Shells (Mactra isabelleana) extracted from Rincón de López $\left(35^{\circ} 46^{\prime}\right.$ $\left.07^{\prime \prime} \mathrm{S}, 57^{\circ} 24^{\prime} 30^{\prime \prime} \mathrm{W}\right)$, Partido de Castelli, from upper part of Destacamento Río Salado Fm. Comment: inner fraction $(80 \%)$ was used.

\section{LP-50. Canal Las Escobas, Sample 1}

$6150 \pm 190$

Shells (Tagelus plebeius) extracted from exposed profile in Las Escobas Channel $\left(35^{\circ} 58^{\prime} 21^{\prime \prime} \mathrm{S}, 57^{\circ} 29^{\prime} 16^{\prime \prime} \mathrm{W}\right)$, Partido de Castelli, Las Escobas $\mathrm{Fm}$, from depth $0.5 \mathrm{~m}$ below surface. Comment: inner fraction $(80 \%)$ was used.

LP-51. Ruta Provincial no. 11, Sample 1

$7890 \pm 340$

Shell (Adelomedon sp) extracted from profile above Ruta Provincial no. 11 ( $35^{\circ} 37^{\prime} 57^{\prime \prime}$ S, $57^{\circ} 18^{\prime} 19^{\prime \prime}$ W), Partido de Magdalena, Las Escobas Fm, from depth $0.4 \mathrm{~m}$ under edaphic sediments. Comment: inner fraction $(80 \%)$ was used.

\section{LP-52. Rincón de López, Sample 3}

$4920 \pm 220$

Shell (Adelomedon brasiliensis) coming from Rincón de López $\left(35^{\circ}\right.$ $46^{\prime} 07^{\prime \prime} \mathrm{S}, 57^{\circ} 24^{\prime} 30^{\prime \prime} \mathrm{W}$ ), Partido de Castelli, extracted from Las Escobas Fm. Comment: inner fraction $(80 \%)$ was used.

\section{LP-54. Manuel B Gonnet}

Modern

Evaporite sample from Calle 516 and Calle $7\left(34^{\circ} 52^{\prime} 28^{\prime \prime} \mathrm{S}, 58^{\circ} 00^{\prime}\right.$ 11" W), Manuel B Gonnet, Particlo de La Plata, extracted from Las Escobas Fm, by sounding from depth $0.5 \mathrm{~m}$ from surface.

\section{LP-66A. Canal Las Escobas, Sample 2 \\ $6490 \pm 230$ \\ LP-66B. \\ $6760 \pm 190$}

Shells (Tagelus plebeius) extracted from exposed profile in Las Escobas Channel ( $35^{\circ} 58^{\prime} 21^{\prime \prime} \mathrm{S}$, $57^{\circ} 29^{\prime} 16^{\prime \prime} \mathrm{W}$ ), Partido de Castelli, Las Escobas Fm from depth $0.5 \mathrm{~m}$ below surface. Comment: for LP-66A, outer fraction $(20 \%)$ was used; for LP-66B, inner fraction $(80 \%)$ was used.

\section{LP-67A. Rincón de López, Sample 4 \\ LP-67B.}

$$
6060 \pm 200
$$

$5930 \pm 220$

Shell (Adelomedon brasiliensis) from Rincón de López (35 $46^{\prime} 07^{\prime \prime}$ $S$, $57^{\circ} 24^{\prime} 30^{\prime \prime} \mathrm{W}$ ), Partido de Castelli, extracted from Las Escobas Fm. Comment: for LP-67A, outer fraction $(20 \%)$ was used; for LP-67B, columella was used. 


\section{LP-68A. Ruta Provincial no. 11, Sample 2}

Shells (Mactra isabelleana) extracted from exposed profile at Ruta Prov no. 11 ( $\left.35^{\circ} 37^{\prime} 57^{\prime \prime} \mathrm{S}, 57^{\circ} 18^{\prime} 19^{\prime \prime} \mathrm{W}\right)$, Partido de Magdalena, Las Escobas $\mathrm{Fm}$, from depth $0.4 \mathrm{~m}$ below edaphic sediments. Comment: for LP-68A, outer fraction $(20 \%)$ was used; for LP-68B, inner fraction was used.

General Comment: (Fidalgo et al, 1981): results from mollusk shells in life position - tanatocenosis - especially LP-50 and -66, yielded ages greater than those determined by other authors for these deposits (Cigliano, 1966; Cortelezzi, 1977). Obtained results from Destacamento Río Salado Fm (LP-49), older than most data from Las Escobas Fm, is consistent with geol relationships between both units: Las Escobas Fm overlies uncomformable Destacamento Río Salado Fm. Evaporite sample (LP-54), with $111 \%$ modern, would indicate contamination by infiltrated meteoric and phreatic waters very close to surface. This result shows age younger than first thermonuclear explosions. X-ray diffraction analyses of samples LP$66 \mathrm{~B},-67 \mathrm{~B}$, and $-68 \mathrm{~B}$ show no evidence of contamination by mineralogic conversion, since these samples are composed of aragonite.

\section{Salina del Bebedero series}

Gastropod shells from ancient shorelines in Salina del Bebedero ( $33^{\circ}$ $20^{\prime} \mathrm{S}, 66^{\circ} 45^{\prime} \mathrm{W}$ ), Prov San Luis (González et al, 1981). Most samples were taken from different outcroppings, except for LP-57 and -58, which come from same profile, at depth of $2 \mathrm{~m}$ and $5.5 \mathrm{~m}$ below surface, respectively. Samples were coll by M A González and taxonomically determined by A Pérez, Univ Nac del Sur (UNS), Bahía Blanca, and D Fernández (FGNyM, UNLP); subm by R Pascual (FCNyM, UNLP). These dates were measured as part of palaeogeog and palaeoclimatic regional study, particularly related to Cordilleran ice ablation from last glaciation to Holocene.

LP-57A. Acceso a Dos Anclas, Sample 1

$15,500 \pm 550$

LP-57B.

$$
17,090 \pm 790
$$

Shells (Chilina parchapei). Comment: for LP-57A, inner fraction $(80 \%)$ was used; for LP-57B, outer fraction $(20 \%)$ was used.

LP-58. Acceso a Dos Anclas, Sample $2 \quad 18,630 \pm 860$ Shells (Chilina parchapei). Comment: inner fraction $(80 \%)$ was used.

LP-59. Puente Arroyo Bebedero

$16,160 \pm 710$ Shells (Chilina parchapei). Comment: inner fraction $(80 \%)$ was used.

LP-60. Sector SO

$\mathbf{1 3 , 9 1 0} \pm \mathbf{5 3 0}$

Shells (Chilina parchapei). Comment: inner fraction (80\%) was used.

LP-61. Camino Dos Anclas-El Lechuzo

$17,060 \pm 660$

Shells (Chilina parchapei). Comment: inner fraction (80\%) was used. 
LP-63. Camino Dos Anclas-Ruta

$15,280 \pm 770$ San Luis/Beazley

Shells (Chilina parchapei). Comment: inner fraction (80\%) was used.

LP-64. Hostería Dos Anclas

$16,950 \pm 870$

Shells (Chilina parchapei). Comment: inner fraction (80\%) was used. General Comment: antiquity of these deposits represents initial ablation of Andean Cordillera ice pertaining to last glaciation (González et al, 1981).

\section{ARCHAEOLOGIC SAMPLES}

LP.53. Arroyo Seco

\section{Argentina}

Collagen extracted from femur fragment of Megatherium americanum, from Arroyo Seco, Partido de Tres Arroyos (38 21 $21^{\prime} 38^{\prime \prime} \mathrm{S}, 60^{\circ} 14^{\prime}$ $39^{\prime \prime}$ W), Prov Buenos Aires. Sample from eolian sediments over carbonate level, from depth $0.6 \mathrm{~m}$ below surface. Coll and subm by $\mathrm{G}$ Politis (FCNyM, UNLP), and L Meo Guzmán (Mus Mun Tres Arroyos). Taxonomic determination by $\mathrm{E}$ Tonni (FCNyM, UNLP). Comment: first determination in Prov Buenos Aires of extinct megamammalian remains in stratigraphic assoc with unifacial lithic material, with marginal retouch, without projectile points.

\section{LP-62. Cuyín Manzano}

$9280 \pm 310$

Charcoal fragments of Cueva Traful I $\left(40^{\circ} 43^{\prime} \mathrm{S}, 71^{\circ} 07^{\prime} \mathrm{W}\right)$, Prov Neuquén, from hearth in Bed $10 \mathrm{~d}$, at depth $1.5 \mathrm{~m}$ below surface. Coll by D Curzio and E Crivelli; subm by M Silveira (all of Fac Filosofía y Letras, Univ Buenos Aires). Comment: absolute age of cultural remains belonging to guanaco hunters with triangular apedunculated projectile points.

\section{GEOCHEMICAL SAMPLES \\ Hydrogeologic samples}

Measurements of environmental isotope content $\left({ }^{3} \mathrm{H},{ }^{2} \mathrm{H},{ }^{18} \mathrm{O}\right.$, and ${ }^{14} \mathrm{C}$ ) were made on meteoric, surface, and underground waters in vicinity of Mar del Plata $\left(38^{\circ} 08^{\prime} \mathrm{S}, 57^{\circ} 35^{\prime} \mathrm{W}\right)$ and surroundings, Prov Buenos Aires (table 1). This study was requested by Cátedra Hidrogeol (FGNyM, UNLP), as part of geohydrol investigation, required by Empresa Obras Sanitarias de la Nación (OSN), Argentina. Aim of study was to formulate series of preliminary hypotheses, related to temporal and spacial origin of groundwaters, and to explain aquifer salinization processes within mentioned area. ${ }^{14} \mathrm{C}$ samples were extracted April 1978 from groundwater at different depths and may belong, in most cases, to more than one productive bed. Results of these measurements are given as percent of modern (\% mod):

$$
\% \bmod =\frac{\text { A sample }-\mathrm{A} \text { std }}{\text { A std }} \times 100 \quad \text { A is net } \mathrm{cpm}
$$


TABle 1

Mar del Plata - ${ }^{14} \mathrm{C}$ measurements in groundwater

\begin{tabular}{ccc}
\hline Sample no. & Well no. (OSN) & $\%$ modern \\
\hline LP-20 & 105 & $71 \pm 1$ \\
-21 & 130 & $79 \pm 1$ \\
-22 & 27 & $72 \pm 2$ \\
-23 & 53 & $83 \pm 1$ \\
-24 & 49 & $85 \pm 1$ \\
-25 & 314 & $94 \pm 1$ \\
-26 & 165 & $75 \pm 1$ \\
-27 & 186 & $60 \pm 1$ \\
-28 & 16 & $88 \pm 1$ \\
-29 & 34 & $70 \pm 1$ \\
-30 & 330 & $112 \pm 1$ \\
-31 & 128 & $91 \pm 1$ \\
-39 & 32 & $102 \pm 3$ \\
-40 & 56 & $88 \pm 2$ \\
-41 & 78 & $79 \pm 2$ \\
-42 & 29 & $70 \pm 2$ \\
-43 & 58 & $73 \pm 1$ \\
-48 & 94 & $63 \pm 1$ \\
\hline
\end{tabular}

General Comment: two groundwater samples (near surface) from "rural" area (LP-25 and -30) show high concentration of ${ }^{14} \mathrm{C}$. This is evidence of atmospheric contamination which started in 1954 and indicates active recharge by meteoric waters in recent years. Other samples, from "urban" area (average depth $90 \mathrm{~m}$ ), were assembled in geog secs:

1) "harbor" (LP-23, -28, -31, -40): measured activity shows recharge by relatively recent rain.

2) "downtown" (LP-22, -29, -41,-42,-43): groundwater of this sec has relatively major residence period and corrected ages should lie between 590 to $1560{ }^{14} \mathrm{C}$ yr.

3) "northern" (LP-20, -21, -26, -27, -48), divided in two sub-secs: S sub-sec shows water mixture of relatively "old" with relatively "young" waters, and N-most sub-sec, with relatively "old" waters, 1000 to $2800{ }^{14} \mathrm{C} \mathrm{yr}$ (Figini et al, 1981).

TABLE 2

${ }^{14} \mathrm{C}$ measurements on atmospheric samples

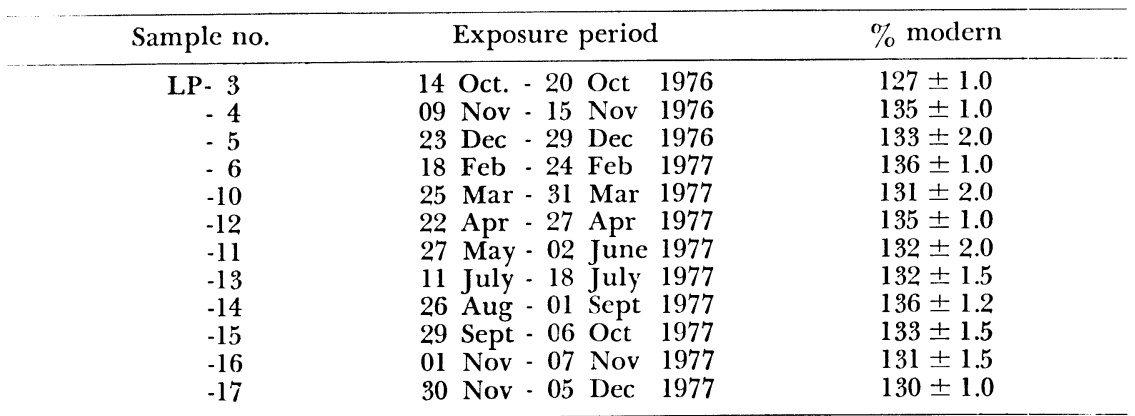




\section{Atmospheric samples}

Determinations were made with samples which cover period, Oct 1976 to Dec 1977, from sampling sta at FCNyM, UNLP (34 ${ }^{\circ} 54^{\prime} 36^{\prime \prime} \mathrm{S}$, $\left.57^{\circ} 56^{\prime} 03^{\prime \prime} \mathrm{W}\right)$, Prov Buenos Aires (table 2).

General Comment: aim of this series was to determine ${ }^{14} \mathrm{C}$ level in atmosphere and influence of nuclear explosions in these latitudes. ${ }^{14} \mathrm{C}$ excess data obtained correspond to theoretical and experimental models of diminution in atmospheric ${ }^{14} \mathrm{C}$ level (Figini et al, 1978).

\section{REFERENCES}

Angulo, R, Fidalgo, F, Gómez Peral, M, and Schnack, E, 1978, Las ingresiones marinas cuaternarias en la Bahía de San Antonio y sus vecindades, Provincia de Río Negro: Cong Geol Argentino, 7th, Actas, Neuquén, v 1, p 271-283.

Cigliano, E, 1966, Contribución a los fechados radiocarbónicos argentinos: Rev Mus La Plata, Antrop, ns, v 6, p 1-16.

Cortelezzi, C, 1977, Datación de las formaciones marinas en el Cuaternario de las proximidades de La Plata-Magdalena, Provincia de Buenos Aires: LEMIT, Anales, v 1.

Fidalgo, F, 1979, Upper Pleistocene-Recent marine deposits in North eastern Buenos Aires Province (Argentina), in Suguio, K, Fairchild, T, Martin, L, and Flexor, J M, eds, Internatl symposium on coastal evolution in the Quaternary, 1978: Sao Paulo,
Brasil, p 384-404.

Fidalgo, F, Figini, A, Gómez, G, Carbonari, J, and Huarte, R, 1980, Algunas dataciones absolutas en sedimentos marinos de la Bahía de San Antonio, Provincia de Río Negro: Simposio problemas geol litoral Atlántico Bonaerense, Mar del Plata: Com Invest Cie Pcia Buenos Aires, Resúmenes, p 243-251.

1981, Dataciones radiocarbónicas en las Formaciones Las Escobas y Destacamento Río Salado, Provincia de Buenos Aires: Cong Geol Argentino, 8th, Actas, San Luis, v 4, p 43-56.

Figini, A, Gómez, G, and Huarte, R, 1978, Variación del carbono 14 en la atmósfera. Su relación con estudios hidrológicos: II reunión informativa Cuaternario Bonaerense, Com Invest Cie Pcia Buenos Aires, Resúmenes, p 29-34.

Figini, A, Gómez, G, Huarte, R, and Carbonari, J, 1981, Aplicación de los isótopos ambientales (carbono 14, tritio, deuterio y oxígeno 18) en las aguas subterráneas del area de la ciudad de Mar del Plata, Provincia de Buenos Aires: Cong Geol Argentino, 8th, Actas, San Luis, v 4, p 21-41.

González, M, Musacchio, E, García, A, Pascual, R, and Corte, A, 1981, Las líneas de costa pleistocenas de la Salina del Bebedero (San Luis, Argentina). Implicancias paleoambientales de sus microfósiles: Cong Geol Argentino, 8th, Actas, San Luis, v 3, p 617-628.

Haynes, C V, 1968, Radiocarbon: analysis of inorganic carbon of fossil bone and enamel: Science, v 161, p 687-688.

Longin, R, 1970, Extraction du collagene des os fossiles pour leur datation par la methode du carbone 14: Thesis no. 553, Univ Lyon, p 1-70. v 230, p 241-242. astro, S, Land, L S, and Varela, A G, 1977, An improved procedure for wet oxidation of the ${ }^{14} \mathrm{C}$ NBS oxalic acid standard: Radiocarbon, v 19, p 375-382.

1979. An improved procedure for wet oxidation of the ${ }^{14} \mathrm{C}$ NBS oxalic acid standard, in Berger, Raincr, and Suess, H E, eds, Radiocarbon dating, Internatl conf on ${ }^{14} \mathrm{C}$ dating, 9th, Proc: Berkeley/Los Angeles, Univ California Press, p 125134

Ward, G K and Wilson, S R, 1978, Procedures for comparing and combining radiocarbon age determinations: a critique: Archaeometry, v 20, no. 1, p 19-31. 